\title{
Combining molecular dynamics and $a b$ initio quantum-chemistry to describe electron transfer reactions in electrochemical environments
}

\author{
David Domínguez-Ariza \\ Departament de Química Física \& Centre Especial de Recerca en Química Teòrica, Universitat de \\ Barcelona \& Parc Científic de Barcelona, C/Martí i Franquès 1, E-08028 Barcelona, Spain \\ Christoph Hartnig \\ Forschungszentrum Juelich IWV 3, D-52425 Juelich, Germany
}

Carmen Sousa and Francesc Illas ${ }^{\text {a) }}$

Departament de Química Física \& Centre Especial de Recerca en Química Teòrica, Universitat de Barcelona \& Parc Científic de Barcelona, C/Martí i Franquès 1, E-08028 Barcelona, Spain

(Received 30 January 2004; accepted 16 April 2004)

\begin{abstract}
A theoretical model is presented aimed to provide a detailed microscopic description of the electron transfer reaction in an electrochemical environment. The present approach is based on the well-known two state model extended by the novelty that the energy of the two states involved in the electron transfer reaction is computed quantum mechanically as a function of the solvent coordinate, as defined in the Marcus theory, and of the intensity of an external electric field. The solvent conformations defining the reaction coordinate are obtained from classical molecular dynamics and then transferred to the quantum mechanical model. The overall approach has been applied to the electron transfer between a chloride anion and a single crystal $\mathrm{Cu}(100)$ electrode. It is found that the solvent exerts a strong influence on the equilibrium geometry of the halide and hence on the relative energy of the two states involved in the electron transfer reaction. Finally, both solvent fluctuations and external field facilitate the electron transfer although solvent effects have a stronger influence. () 2004 American Institute of Physics. [DOI: 10.1063/1.1760071]
\end{abstract}

\section{INTRODUCTION}

Electron transfer (ET) reactions in homogeneous phase or at heterogeneous interfaces play a central role in many processes in chemical ${ }^{1,2}$ or biological ${ }^{3}$ systems. Electron transfer at electrode surfaces constitutes one of the main subjects of Electrochemistry and hence has been studied extensively both by experimental and theoretical approaches. ${ }^{1}$ The first theoretical studies of electron transfer reactions are due to Marcus, ${ }^{4,5}$ who proposed a semiclassical model and showed the important role of the solvent fluctuations in the ET reactions. Later Levich and Dogonadze ${ }^{6}$ and Marcus ${ }^{7}$ refined the theories and included the quantum nature of the metal surface. Recently, classical molecular dynamics (MD) simulations were used to explain deviations with respect to the Marcus predictions. ${ }^{8-10}$ More refined models have been presented in the framework of the Anderson-Newns Hamiltonian for chemisorption, ${ }^{11,12}$ which accounts for the quantum nature of the process. ${ }^{13-15}$ These approaches have been extended by including the effect of the presence of counterions and of the redox ions mobility ${ }^{16}$ and also by taking into account the polarizability of the solvent. ${ }^{17}$ The development of different theories of electron transfer reactions has been recently reviewed by Hush. ${ }^{18}$

The huge complexity of the electrochemical environment is the main bottleneck when attempting to propose theoretical models for these reactions. In principle, a full de-

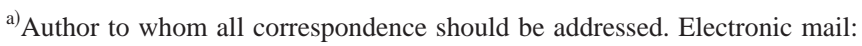
francesc.illas@ub.edu
}

scription of the ET reactions would require one to take into account the solvent structure, its interaction with the electrode and with the solute, the interaction of the solute with the metallic surface, the electronic structure of the electrode, the ionic force, etc. Because of this complexity most of the ET models commented on above are essentially phenomenological, they are based on a given model Hamiltonian and their predictions depend on a series of parameters that are usually obtained by means of experiments or ab initio calculations. Notwithstanding this tremendous complexity, many attempts to describe ET reactions at electrodes by means of quantum chemical methods have been reported. Most of these studies use the cluster model approach to represent the electrode surface, and a variety of quantum chemical methods, from simple extended Hückel calculations to rather sophisticated $a b$ initio methods, including Hartree-Fock and density functional theory approaches, is applied. ${ }^{19-25}$ However, in most of these studies only the electronic ground state is considered, whereas the ET reaction involves at least two electronic states, ${ }^{1,2}$ one of them with the transferred electron located on the electrode, and the other one with this electron located on the redox species. On the other hand, the effects of the electrode potential are usually not included or represented only in a rather crude way.

In a recent work, ${ }^{25}$ a model based on the application of ab initio quantum chemical methods was presented with the aim of including two aspects that were usually not taken into account in previous works. Namely, the effect of the external electric field due to the electrode potential and the presence 
of more than one electronic state. The model was based on the two states approximation for ET reactions, ${ }^{2}$ and thus two different constituents could be identified in the system, those are the donor $(D)$ and the acceptor $(A)$. It was assumed that two electronic states contribute mainly to the ET process, those are the electronic states prior $(D A)$ and after $\left(D^{+} A^{-}\right)$ the ET reaction. These two electronic states can be regarded as two resonating forms in the valence bond sense and the ET reaction would occur when these two states become degenerate. ${ }^{25}$ To handle these electronic states simultaneously a multiconfigurational description was chosen of the corresponding wave functions dominated by the $D A$ or $D^{+} A^{-}$valence bond configurations with the concomitant requirement to use a cluster model to describe the electrode surface. Finally, the effects of the electrode potential were included by adding a uniform external electric field oriented perpendicular to the electrode surface. The orientation of the electric field determines the relative stabilization of the $D A$ and $D^{+} A^{-}$states. The model was successfully applied to the gas-phase oxidation of halides on a $\mathrm{Cu}_{25}$ cluster model of the electrode. ${ }^{25}$ The halide and electrode surface are the donor and acceptor, respectively; hence $\mathrm{Cu}_{25} \mathrm{X}^{-}(A D)$ and $\mathrm{Cu}_{25}^{-} \mathrm{X}$ $\left(A^{-} D^{+}\right)$are the electronic states involved in the ET reaction. The relative stability of these two electronic states was studied as a function of the intensity of an external uniform electric field and solvent effects were neglected. For a given value of the electric field intensity, $f_{0}$, the two states become degenerate and the electron transfer reaction can proceed spontaneously and radiationless. For each halide a different $f_{0}$ threshold value is found. In fact, a linear dependence between $f_{0}$ and the standard equilibrium $\mathrm{X} / \mathrm{X}^{-}$red-ox potential is observed which indicates that the model is able to capture the essential physics of halide oxidation. ${ }^{25}$

However, in spite of these encouraging results, the proposed model has still an important drawback, the absence of solvent molecules. These effects are crucial to describe the ET reaction in an electrochemical environment. The lack of solvent effects may explain that the $f_{0}$ values are significantly larger than typical values in electrochemical cells. Recently, Hartnig and Koper ${ }^{26}$ presented a study of the solvent reorganization role in the oxidation reaction of halides on electrodes. This study is based on the framework of the Marcus theory using classical MD simulations. The authors explicitly considered the solvent contributions to the reaction but neglected the electrode electric field effects. The two contributions described above ${ }^{25,26}$ represent complementary approaches to ET reaction in an electrochemical environment. Clearly, a theoretical framework combining these two strategies is highly desirable and is the main goal of the present work. To this end, we present a new theoretical model for ET reactions at electrode surfaces simultaneously including the presence of two electronic states, the electrode potential and solvent effects. The electrode potential is modeled by a uniform external electric field whereas solvent effects are taken into account by using classical molecular dynamics. For the pertinent geometrical arrangements, a large number of configurations of water molecules surrounding the chloride anion has been obtained from classical MD simulations in the presence of the external electric field when nec- essary. Next, the electrostatic solvent effects have been incorporated into the $a b$ initio quantum chemical treatment. This is justified since the main contribution to the interaction between an ion and the solvating water molecules is of electrostatic origin. To the best of the authors' knowledge, this is the first attempt to describe ET at electrodes using $a b$ initio quantum chemical techniques and including all the aforementioned factors. This new approach has been applied to the oxidation of a chloride anion next to a copper electrode in aqueous solution.

\section{COMPUTATION}

\section{A. Molecular dynamics simulation}

The MD simulations of the solvated redox species next to a copper surface were performed in a rectangular simulation box containing 440 water molecules and one solute species with a confining wall potential at one side of the unit cell. The side length of the quadratic basis parallel to the surface is $20.4478 \AA$. The confinement due to the electrode is modeled by a (100) copper surface $(8 \times 8$ atoms wide) built of four layers of copper atoms. A confining potential with the repulsive part of the 9-3 Lennard-Jones potential acting on the oxygen atoms was applied parallel to the metal surface to prevent water molecules from evaporating; the parameters of this potential were chosen to achieve bulk density in the center of the liquid phase. Two-dimensional periodic boundary conditions were chosen along the surface.

The rigid SPC/E potential was used to model the solvent. ${ }^{27}$ Studies on ET processes on electrodes including solvent polarizability have shown that there is a significant effect of solvent polarizability on the free energy curves of the redox process. However, it is also shown that the effect of solvent polarizability on the distribution of the solvent molecules around the redox center and on the electrode is almost negligible. ${ }^{17}$ This is precisely the information obtained in the present MD simulations which is used in a subsequent step to introduce, albeit in an approximate way, the solvent influence in the ET reaction. Therefore, the use of the SPC/E model, which does not include the polarizability of the water molecules, is adequate enough. Interactions between water molecules and the metal atoms were taken from literature. ${ }^{28,29}$ The solute species was described as a soft sphere bearing a singly negative or no charge in the center, depending on the considered state. The LennardJones parameters for the ion-water interactions are representative for the halogen $\mathrm{Cl}^{30}$ Since in each case the solute position is fixed, no interactions of the redox species with the metallic surface are calculated. Long-ranged electrostatic forces were calculated using a three-dimensional-Ewald summation with corrections for two-dimensional periodic geometry. ${ }^{31}$ A partial charge transfer (PCT) between the solute and the electrode was neglected to obtain the configurations of the solvation shell. Previous studies including the PCT by means of the Anderson-Newns approach showed that even for a contact adsorbed chloride on a platinum electrode the transferred charge amounted to $0.06 e$ only (see Ref. 26 for further details).

The temperature was held constant at $298.15 \mathrm{~K}$ using a 
Nose-Hoover thermostat. Newton's equations of motion were integrated using the Verlet algorithm with a time step of $1 \mathrm{fs}$. The constraints for the rigid water model were fulfilled using the SHAKE algorithm.

\section{B. The solvent coordinate}

To investigate solvent effects on the relative energy of the two electronic states involved in the ab initio ET model, ${ }^{25}$ a variety of models with different distances of the redox species to the electrode surface have been considered. The systems were prepared by setting the solute species on a fixed distance from the (100) copper surface in a fourfold hollow position. After an equilibration period of up to $50 \mathrm{ps}$ the procedure went on as follows: The equilibrium configuration of the solvation shell was identified following the principles of the Marcus theory. ${ }^{4,5,7}$ The multidimensional description of the solvating molecules is next mapped to one variable, the so-called solvent coordinate $\Delta E$. Around the equilibrium position, fluctuations of the solvent coordinate show a parabolic behavior. Fitting a parabola to this probability distribution permits one to find the equilibrium value, $\Delta E_{0}$, of $\Delta E$. The equilibrium solvent coordinate $\Delta E_{0}$ varies between 0 and 1 , the two limits represent the effect of the solvent average equilibrium configuration around $\mathrm{Cl}^{0}$ and $\mathrm{Cl}^{-}$, respectively (for further computational details see Refs. 8 and 15, and references therein). For successive quantum mechanical calculations, representative configurations leading to the equilibrium value of $\Delta E_{0}$ for $\mathrm{Cl}^{0}$ and $\mathrm{Cl}^{-}$were chosen and molecules within a box with a quadratic basis with a side length of $10.224 \AA$ [resembling the dimension of a four atoms wide (100) $\mathrm{Cu}$ surface] were considered. For calculations with varying values of $\Delta E_{0}$, a linear interpolation between the two extreme values was chosen.

\section{C. $A b$ initio model for the oxidation of solvated chloride on the $\mathrm{Cu}(100)$ electrode}

The application of the $a b$ initio quantum chemical methods to the oxidation of the halide on the copper electrode has been done following the model recently proposed in Ref. 25 . This model is based on the two states donor-acceptor model for intra- and intermolecular ET reactions ${ }^{2}$ and is extended here to nonspecifically adsorbed species. The solvent effect in the reaction has been included by adding explicitly the contribution of the water molecules representing a given value of the solvent coordinate in the MD simulations. However, to make the calculations feasible the water molecules are replaced by point charges; $q(\mathrm{O})=-0.8476, q(\mathrm{H})$ $=0.4238$ which are the values used in the potentials employed to carry out the MD calculations. While more sophisticated treatments of electron transfer in solution have been reported recently, ${ }^{10}$ the present choice is a compromise between accuracy and feasibility. In fact, the present model still permits a fully $a b$ initio approach to the two-state model although it has the limitation of not including the electronic interactions between the water molecules and the metallic surface. In this respect, $a b$ initio molecular dynamics studies on the $\mathrm{Cu}(110)$-water ${ }^{32}$ and $\mathrm{Ag}(111)$-water ${ }^{33}$ interfaces have shown evidence of a coupling between the electronic states

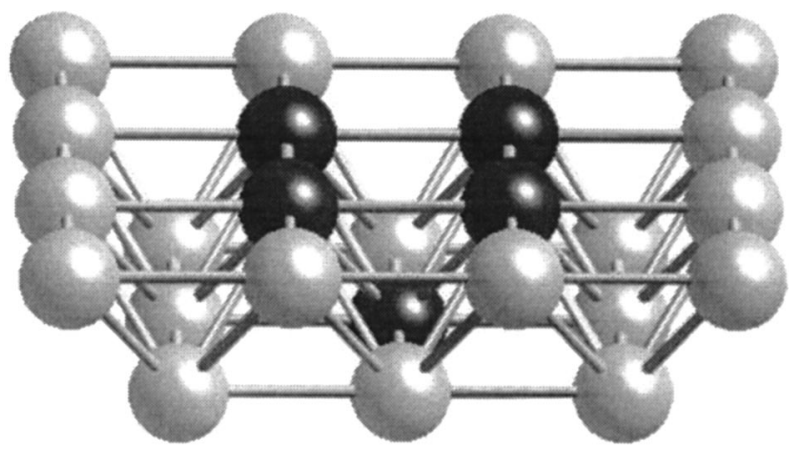

FIG. 1. Cluster model representation of the $\mathrm{Cu}(100)$ surface used to study chloride adsorption on the fourfold hollow position. Dark atoms belong to the local region and light atoms belong to the outer region.

of the solid and those of the water system, but also show that surface electronic states remain almost unaffected by the presence of water molecules. Since in the present $a b$ initio two-state electron transfer model used it is assumed that ET takes place as a consequence of the interaction between one surface electronic state of the metal and one electronic state of the redox center, the neglect of the electronic interactions between the electrode and the solvent is, at least to a firstorder approximation, justified. On the other hand, the interactions between the solute (chloride) and the electrode are entirely included in the model, since both parts of the system are treated fully $a b$ initio. Thus, the partial charge transfer effects between the solute and the electrode are also accounted for. A cluster model containing 25 copper atoms (16 forming the first layer, 9 the second one) has been used to model a $\mathrm{Cu}(100)$ surface (Fig. 1). Two regions have been distinguished, the local and the outer region. The first one contains the five central atoms of the cluster around the fourfold hollow position (see Fig. 1). These $\mathrm{Cu}$ atoms were described with the small core effective core potential reported by Hay and Wadt; ${ }^{34}$ the corresponding double- $\zeta$ basis set being used to describe the outermost 19 electrons. The external region contains the 20 remaining atoms, which have been described as one-electron pseudoatoms ${ }^{35}$ and thus only the $4 s$ electron of each $\mathrm{Cu}$ atom has been explicitly considered with a $(4 s 1 p / 2 s 1 p)$ basis set. The $\mathrm{Cu}-\mathrm{Cu}$ distance has been fixed to be the experimental bulk value $(2.556 \AA)$. The chloride anion has been placed on the fourfold hollow position at different distances to the surface, from the equilibrium position in ultrahigh-vacuum (UHV) conditions $(1.99 \AA)^{25}$ up to $6.0 \AA$. For the chloride ion, an atomic natural orbital basis set has been used, contracted to a double- $\zeta$ plus polarization level. ${ }^{36}$

The $a b$ initio quantum chemical calculations have been done at the open shell restricted Hartree-Fock (OSRHF) or at the complete active space configuration interaction (CAS-CI) level. The OSRHF calculations were performed to study the systems only at their electronic ground state, whereas the CAS-CI method was used to account simultaneously for two electronic states matching precisely the two possible oxidation states of the chlorine. The use of a multireference $N$-electron wave function permits the simultaneous description of the two electronic states involved in the ET 
process and to take into account explicitly the electronic coupling between them. Therefore, there is no approximation with respect to the adiabaticity of the process. The active space in the CAS-CI calculations contained the minimum orbital space necessary to describe the electronic states under consideration $\left(\mathrm{Cu}_{25} \mathrm{Cl}^{-}\right.$and $\left.\mathrm{Cu}_{25}^{-} \mathrm{Cl}\right) .{ }^{25}$ Hence, it included the $\mathrm{Cl} 3 p$ orbital perpendicular to the surface and the singly occupied molecular orbital of the cluster modeling the electrode, for a total of three electrons.

The presence of the electrode potential will influence the relative position of the two electronic states so that one should compute the variation of the energy of each state $\left(E_{1}\right.$ and $E_{2}$ ) with respect to the electrode potential $V, \partial E_{i} / \partial V$. However, $\partial E_{i} / \partial V=\left(\partial E_{i} / \partial F\right)(\partial F / \partial V)$ and for a uniform external electric field $F$ one has indeed $\partial F / \partial V=C / \varepsilon$ $=d_{\mathrm{DL}}$, where $C$ is the capacity of a parallel plate condenser model separated at a distance, $\varepsilon$ is the medium electric permittivity, and $d_{\mathrm{DL}}$ represents the electronic double layer thickness. $^{37,38}$ Therefore, the effects of the external potential on $E_{1}$ and $E_{2}$ can be represented by adding a uniform external electric field to the exact nonrelativistic Hamiltonian. Consequently, the energy of the two electronic states was obtained as a function of the external field intensity and of the solvent coordinate.

The $a b$ initio quantum chemical calculations have been performed with the MOLCAS-5 software package. ${ }^{39}$ Notice that introducing the water molecules in the calculations, albeit in an approximate way, removes all symmetry operations.

\section{RESULTS}

\section{A. Influence of the solvent on the equilibrium geometry of the adsorbed chloride anion}

The first application of the $a b$ initio model to chargetransfer at electrodes neglected the solvent effects and considered the equilibrium geometry of different halides under ultrahigh-vacuum conditions. ${ }^{25}$ One of the possible solvent effects is precisely the change on the equilibrium distance of adsorbed species. In fact, previous works have shown that for $\mathrm{Fe}^{3+},{ }^{16}$ and also for $\mathrm{Na}^{+}$and $\mathrm{Cl}^{-},{ }^{40}$ the equilibrium distance from the solvated ion to the metallic electrode is approximately $5 \AA$, considerably larger than the value expected for the UHV conditions. Therefore, before applying the two state model for ET to the present case, it is necessary to obtain the equilibrium position of the chloride anion at the metal-electrolyte interface. The search for the equilibrium geometry has been carried out at the OSRHF level of theory. The OSRHF energy has been computed for various distances from the chloride anion to the electrode surface and including the electrostatic effect of the water molecules surrounding the anion explicitly. For each distance, this is achieved by including the point charge representation of the water molecules described above for the configurations obtained from the classical dynamics simulations. The corresponding potential energy curve is reported in Fig. 2. This figure also reports the corresponding potential energy curve for the interaction in absence of the water molecules and the difference between these two curves. The curve including the ef-

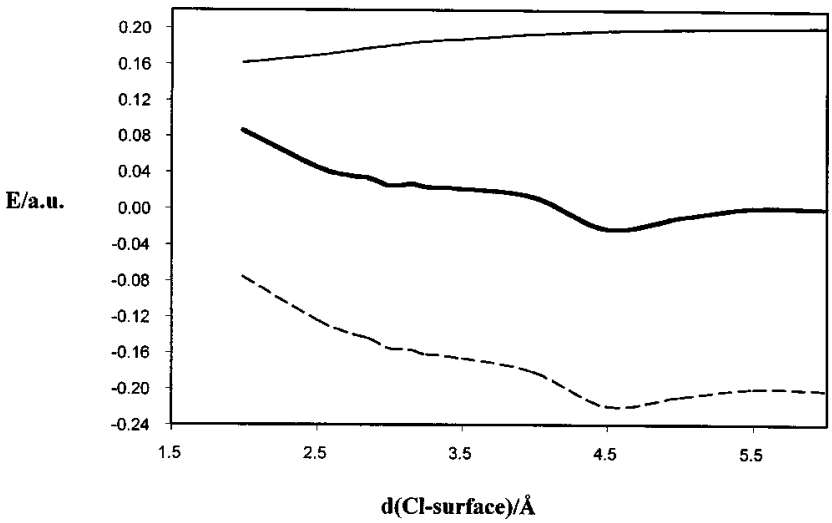

FIG. 2. Potential energy curve of the $\mathrm{Cu}_{25} \mathrm{Cl}^{-}$model system including the effects of the water molecules (-), potential energy curve of the $\mathrm{Cu}_{25} \mathrm{Cl}^{-}$ model system in the gas phase (-), and the difference between them (---). The zero energy corresponds to the energy of the surface and the solvated anion at large distance.

fect of the water molecules shows a minimum at around 4.5 $\AA$ and becomes slightly repulsive in the $2.0 \AA$ region, close to the point where the minimum energy in the absence of solvent is found. ${ }^{25}$ Qualitatively, this curve is in agreement with the prediction of Ignaczak et al. ${ }^{28}$ although these authors also find a shallow minimum at a shorter distance from the surface. Nevertheless, this feature is strongly dependent on the potential used in their Monte Carlo simulations, and the important message is that the presence of the solvent leads to a significant increase in the equilibrium distance from the adsorbate to the surface.

There are two opposite forces that are responsible for the observed behavior, namely the adsorbate-surface and the adsorbate-solvent interactions. The adsorbate-surface interaction has an attractive region with a pronounced minimum. In absence of solvent this interaction is responsible for the relatively short equilibrium distance. However, there is also an electrostatic attractive interaction between the solvent and the chloride anion. This interaction depends directly on the number of water molecules in the ion solvation shell. At large distances from the electrode, the solvation sphere is complete, and hence the solvent-halide interaction attains its maximum. At shorter distances, the water molecules cannot completely solvate the anion, which implies a destabilization of the system. The competition between these two interactions leads to an equilibrium distance of $\sim 4.5 \AA$, longer than that found without solvent, but shorter than "infinite" which would be expected if there were no attractive interactions between the electrode and the anion.

The equilibrium position of the solvated anion at a distance of $\sim 4.5 \AA$ can also be explained from a more qualitative point of view. The oxygen atoms density distribution (solid line in Fig. 3) shows a distinct layering of the solvent with a first peak around $2.2 \AA$ and a second one at $\sim 5 \AA$, a behavior also observed in previous studies. ${ }^{15,16,26,40}$ Therefore, at a distance from the surface of $\sim 4.5 \AA$ the ion still feels the attraction from the electrode but can also keep its complete hydration shell formed by solvent molecules from the first layer as well as molecules from the next layers. A shorter distance to the electrode surface would not only im- 


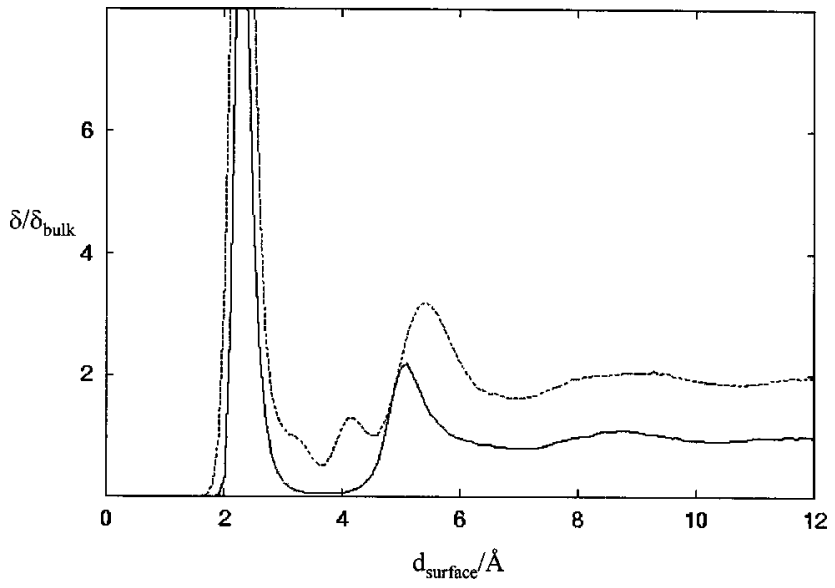

FIG. 3. Density distribution of the oxygen (-) and hydrogen (---) atoms of the water molecules relative to the bulk density as a function of the distance to the electrode surface as obtained from classical molecular dynamics simulations.

ply a partial desolvation but also desorption of at least one water molecule that is adsorbed on the surface. From an entropic point of view, the partial loss of the solvation shell of the specifically adsorbed halide ion is favored; however the energy cost is very large.

In the case of using approximate Hamiltonians, it has been reported that ion and solvent image effects can be responsible for an increase of the computed equilibrium distance of up to $10 \% .{ }^{16}$ However, in the present model the electronic structure of the electrode has been explicitly included in the OSRHF calculations, and hence the response of the electrode to the presence of a distribution of electric charges is present. In fact, an accurate analysis of the image charge effects shows that at intermediate distances of the metal surface, similar to those involving the solvated $\mathrm{Cl}$ anion, the response of the metal surface is well represented by a Hartree-Fock cluster model approach. ${ }^{41}$

Therefore, one can conclude that in the vicinity of the copper electrode, the chloride anion resides most likely at a distance of about $4.5 \AA$ from the surface, whereas the possibility of finding it adsorbed on the surface, after a partial loss of the solvation sphere, is not negligible. This is an important conclusion, since it determines the position of the chloride for further studies of the effects of solvent and the electric field on the ET reaction.

\section{B. Influence of the solvent on the electron transfer reaction}

Once the equilibrium position of the halide has been estimated, one can use the two-state model to study the influence of the solvent fluctuations on the energy of each one of the electronic states involved in the ET. To this end, $\mathrm{CAS}-\mathrm{Cl}$ calculations were carried out to determine the energy of the $\mathrm{Cu}_{25} \mathrm{Cl}^{-}$and $\mathrm{Cu}_{25}^{-} \mathrm{Cl}$ states for different values of the solvent coordinate and with the $\mathrm{Cl}$ nucleus placed at 4.5 $\AA$ from the surface on the fourfold hollow position. The different points along the solvent coordinate have been modeled by using the appropriate solvent configurations (see Sec. II); the results are summarized in Fig. 4.

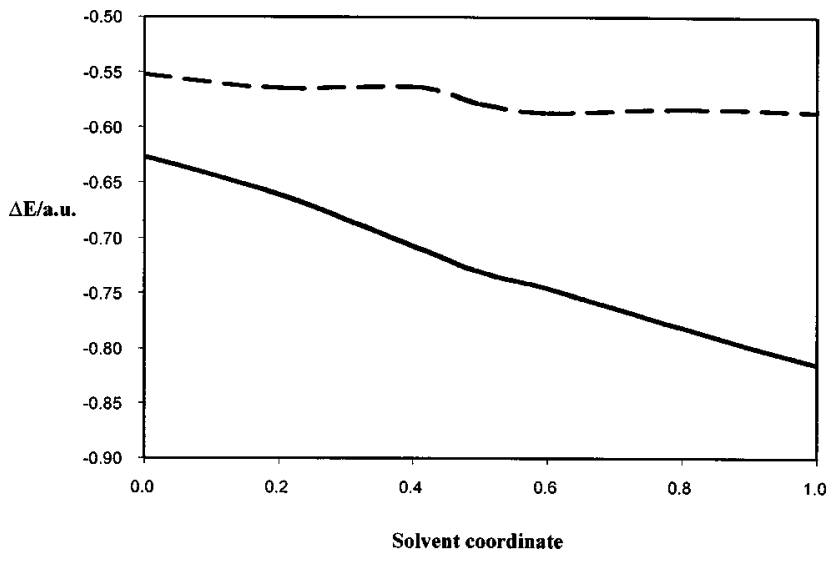

FIG. 4. Total energy of the $\mathrm{Cu}_{25} \mathrm{Cl}^{-}$(-) and $\mathrm{Cu}_{25}^{-} \mathrm{Cl}$ (---) electronic states vs the solvent coordinate computed at the CAS-CI level of theory. The $\mathrm{Cl}$ nucleus is placed at $4.5 \AA$ from the surface. 1439 a.u. have been added to the energy values represented in the $y$ axis.

The first important observation is the marked different dependence of the energy of each electronic state with respect to the solvent coordinate. The energy of the chloride state $\left(\mathrm{Cu}_{25} \mathrm{Cl}^{-}\right)$exhibits an almost linear decrease when going from 0.0 to 1.0 in the solvent coordinate. However, the energy of the chlorine state $\left(\mathrm{Cu}_{25}^{-} \mathrm{Cl}\right)$ seems to be almost constant along the solvent coordinate and, hence, much less affected by solvent fluctuations. This different behavior is not surprising and it is mainly due to the electrostatic nature of the interactions involved. The chloride anion bearing a negative electric charge is very sensitive to fluctuations of the solvent around it and the opposite holds for the chlorine atom. The later has no net electric charge and its interactions with the solvent are limited to mainly weak interactions (polarization and dispersion), only partially included in our model and, in any case, much smaller than those involved between the chloride anion and the solvent. This behavior is also related to the dependence of the solvent reorganization energy with respect to the distance to the electrode observed for both the anion and the atom. ${ }^{26}$

The different influence of the solvent fluctuations on the electronic states under study exerts an important effect on the possibility of a successful electron transfer. Figure 4 shows that the energy difference between the two electronic states is very large — $\sim 6.2 \mathrm{eV}$-for the 1.0 solvent coordinate, which precisely corresponds to ideal anion solvation. This energy difference decreases to $\sim 2.0 \mathrm{eV}$ when going to the solvent coordinate 0.0 . This implies that the electronic state corresponding to the neutral chlorine atom is stabilized by $\sim 4.2$ $\mathrm{eV}$ with respect to the chloride anion state by the solvent fluctuations. However, in spite of this strong stabilization the order of the electronic state is not reversed meaning that, in the absence of an external potential, $\mathrm{Cl}^{-}$is not spontaneously oxidized, or, in turn, a hypothetical $\mathrm{Cl}^{0}$ close to a copper electrode will be reduced immediately. This is obviously in agreement with the observed experimental behavior.

\section{Influence of the equilibrium position on the electron transfer reaction}

The position of the redox center with respect to the electrode is known to have an important effect on the electron 


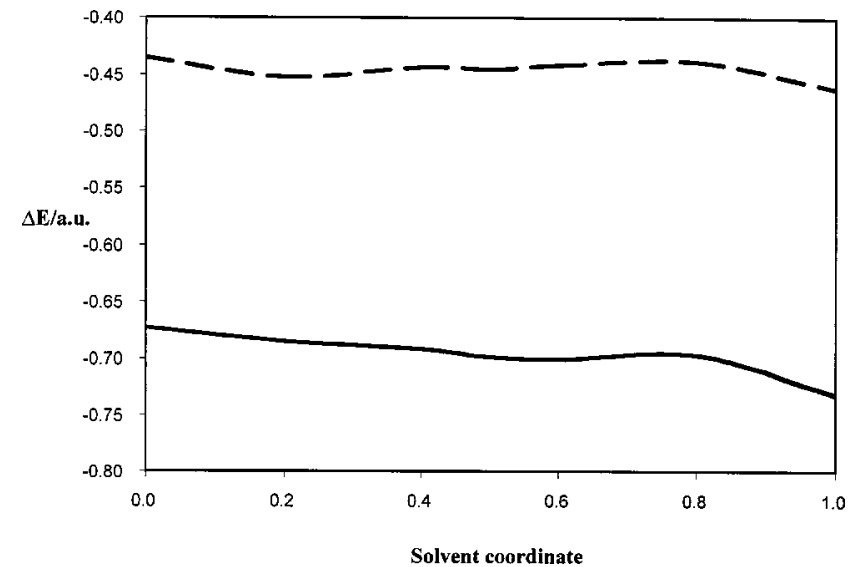

FIG. 5. Same as Fig. 4 but in this case the $\mathrm{Cl}$ nucleus is placed at $1.99 \AA$ from the surface. 1439 a.u. have been added to the energy values represented in the $y$ axis.

transfer reaction. These effects have been studied in detail for the $\mathrm{Fe}^{3+} / \mathrm{Fe}^{2+}$ process. ${ }^{16}$ In this part we turn our attention to the dependence of the energy difference between the two electronic states involved in the ET reaction on the distance of the anion to the electrode surface. Note that according to the $a b$ initio two state model, ${ }^{25}$ the smaller this energy difference, the smaller would be the electric field needed to trigger the ET reaction. To determine the influence of the distance from the adsorbate to the surface, the energy of the two electronic states involved in the ET reaction has been computed again as a function of the solvent coordinate (Fig. 5) but placing the $\mathrm{Cl}$ species at $1.99 \AA$ which is the equilibrium distance for $\mathrm{Cl}$ on $\mathrm{Cu}(100)$ under UHV conditions. ${ }^{25}$ Comparison of Figs. 4 and 5 reveals a qualitative different behavior of the electronic state related to the $\mathrm{Cu}_{25} \mathrm{Cl}^{-}$form. As stated earlier, when the $\mathrm{Cl}$ nucleus is at $4.5 \AA$ from the surface, the energy of this electronic state exhibits a nearly linear dependence with respect to the solvent coordinate. This is not the case when the $\mathrm{Cl}$ nucleus is placed at $1.99 \AA$ above the surface. In this case the total energy of this electronic state does only show a small decrease when going from 0.0 to 1.0 along the solvent coordinate. The reason for this result is rather simple, at a short distance from the surface, the anion has lost at least half of its solvation sphere and, as a consequence, the solvent effects are smaller, whereas the interaction with the electrode is more relevant. This is also reflected in the energy difference between the $\mathrm{Cu}_{25} \mathrm{Cl}^{-}$and $\mathrm{Cu}_{25}^{-} \mathrm{Cl}$ electronic states. When the halide is placed at $1.99 \AA$ above the surface, this energy difference changes from 7.3 to $6.5 \mathrm{eV}$ when going from 1.0 to 0.0 in the solvent coordinate. This means that in this case the solvent fluctuations can stabilize the chlorine state with respect to the chloride one but only by $0.8 \mathrm{eV}$. This is five times smaller than the stabilization observed when the halide is placed at $4.5 \AA$ above the surface. Therefore, for shorter distances where the halogen is specifically adsorbed, the solvent effects are smaller than at longer distances, where the solvation sphere around the ion is complete. As a consequence, at shorter distances to the electrode, the induction of the electron transfer process by the solvent fluctuations is even less feasible than at longer distances.

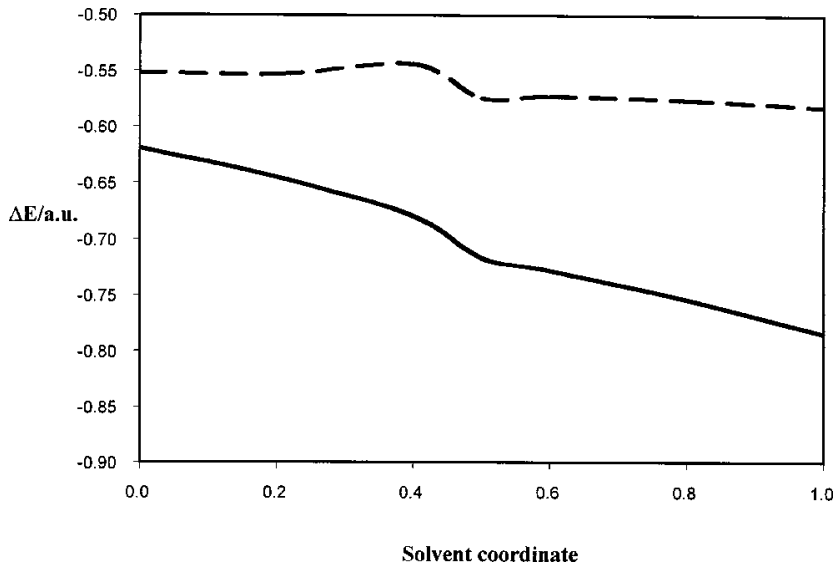

FIG. 6. Energy of the $\mathrm{Cu}_{25} \mathrm{Cl}^{-}$(-) and $\mathrm{Cu}_{25}^{-} \mathrm{Cl}$ (---) electronic states vs the solvent coordinate computed at the CAS-CI level of theory under the presence of an uniform external electric field with an intensity of $10^{9} \mathrm{~V} / \mathrm{m}$ (see the text). The anion is placed at $4.5 \AA$ from the surface. 1439 a.u. have been added to the energy values represented in the $y$ axis.

\section{Influence of an external electric field on the electron transfer reaction}

Finally, we comment on the results regarding the influence of the electrode electric field on the ET process. To this end, a new series of calculations, analogous to those discussed in Sec. III B, has been carried out in which the presence of a uniform external electric field perpendicular to the electrode has been included explicitly (see Sec. II). The intensity of the field is $10^{9} \mathrm{~V} / \mathrm{m}$, which is of the order of electric fields in the electric double layer; the field is oriented in such a way that it models an anodic process (the field vector points toward the solution side). Here, it is important to remark that the electric field has been included in the $a b$ initio calculations and also in the molecular dynamics simulations. This implies that, for each value of the external electric field, the water molecules configurations employed in the ab initio model have been obtained under the presence of the same electric field. The dependence of the energy of the two electronic states involved in the ET reaction with respect to the solvent coordinate under the presence of the electric field is shown in Fig. 6. The overall behavior is quite similar to that described in Sec. III A. Here, however, a bending in the curves is observed when the solvent coordinate becomes 0.5. Although there is no clear explanation for this behavior it is likely that it arises from the statistical nature of the choice of the water configurations. Thus, for this particular value of the solvent coordinate the results may appear to be more approximated in nature. The $\mathrm{Cu}_{25} \mathrm{Cl}^{-}$state is again stabilized almost linearly when going from 0.0 to 1.0 along the solvent coordinate, whereas the $\mathrm{Cu}_{25}^{-} \mathrm{Cl}$ state does only show a very weak dependence, with respect to the solvent coordinate. However, the important point is that the presence of the external electric field provokes an additional reduction of the energy difference between the two states under study. In fact, for the solvent coordinate $1.0\left(\mathrm{Cl}^{-}\right)$the energy difference is $5.5 \mathrm{eV}$ in presence of the electric field whereas otherwise it is $6.2 \mathrm{eV}$. For the solvent coordinate $0.0\left(\mathrm{Cl}^{0}\right)$, the energy difference is $1.9 \mathrm{eV}$ whereas it is $2.0 \mathrm{eV}$ without including the 
external electric field. Hence, the external electric field is responsible for a decrease of $\sim 0.1-0.7 \mathrm{eV}$ in the energy difference.

To explain the stronger effect of the electric field on the energy differences at the solvent coordinate 1.0 one has to examine the interactions incorporated in the system due to the inclusion of the field. The first one is the interaction of the electric field with the negative charge of the halide anion, which is pushed toward the electrode. Obviously, this interaction is missing in the $\mathrm{Cu}_{25}^{-} \mathrm{Cl}$ state, and this is the main reason for the reduction of the energy difference between the two states. A second effect corresponds to the distortions of the optimal solvation structures around the halide or the halogen due to the interactions of the water molecules with the external electric field. When the external electric field is applied, with the solvent coordinate 1.0 the $\mathrm{Cu}_{25} \mathrm{Cl}^{-}$state is destabilized by the two factors commented above. On the other hand, the effect in the energy of the $\mathrm{Cu}_{25}^{-} \mathrm{Cl}$ is smaller because this system is quite insensitive to solvent fluctuations. At the solvent coordinate 0.0 , the $\mathrm{Cu}_{25} \mathrm{Cl}^{-}$state is mainly destabilized due to the interaction of the field with the anion. Since now the solvent structure corresponds to the uncharged atom and is therefore not optimal for the halide anion, the distortions of the solvent structure due to the presence of the electric field will have only a small influence on the energy of this state. Summarizing, the stronger destabilization of the $\mathrm{Cu}_{25} \mathrm{Cl}^{-}$state in the solvent coordinate 1.0 is caused by the distortions of the solvent structure due to the electric field effects, which have smaller effects at the solvent coordinate 0.0 .

Electric fields of stronger intensity are expected to diminish the difference between the states involved in the process even more and up to the point where the two states become degenerate. ${ }^{25}$ However, from these results it is clear that the solvent fluctuations have a stronger influence on the relative stability of the electronic states involved in the ET process than the electrode electric field in agreement with previous works. ${ }^{26,42}$ Notice that the electrode electric fields present in electrochemical systems have intensities similar to that used in this study. The use of electric fields of much higher intensity is not appropriate to adequately represent an electrochemical system.

\section{CONCLUSIONS}

The electron transfer process between a chloride anion and a copper electrode surface has been studied by combining classical molecular dynamics and ab initio quantumchemistry methods. Molecular dynamics simulations have been used to reproduce the role of the solvent fluctuations during the process. The water configurations obtained have been taken into account for the electrostatic contribution of the solvent effects in the quantum chemical calculations. This rather simple solvent representation is able to reproduce most of the solute-solvent interactions.

Incorporating the solvent effects has important consequences: First, there is a very large change on the optimum adsorbate-surface distance with respect to that corresponding to ultrahigh-vacuum conditions. This is due to the competi- tion between the need to preserve the solvation sphere and the attractive interactions with the metal surface. However, the possibility of finding the redox species at closer distances cannot be neglected due to entropic effects. Second, the solvent fluctuations taken into account by the proper definition of the solvent coordinate exert a differential effect on the two electronic states involved in the electron transfer reaction. This effect is significantly larger when the $\mathrm{Cl}$ nucleus is at a distance from the surface close to the equilibrium value in solution and decreases when the anion is located at shorter distances due to the partial loss of the solvation sphere. Finally, the presence of an external electric field preferentially stabilizes the oxidized $\left(\mathrm{Cu}_{25}^{-} \mathrm{Cl}\right)$ state, although its effect is weaker $(\sim 0.5 \mathrm{eV})$ than the effect exerted by the solvent fluctuations.

The results obtained in the present work indicate that the discharge of the chloride on the copper electrode takes place as an ion transfer reaction. This comes from the fact that an electron transfer reaction would imply a rather short distance between the anion and the metal surface. However, when the anion approaches the surface, the coupling between the electronic states involved in the reaction increases and then the direct electron transfer process is favored. Moreover, to approach the halide to the electrode it is necessary to surmount an energy barrier. This can be considered as a "desolvation energy" which has to be overcome to partially shed off the solvation shell (Fig. 2). Hence, while at distance of $\sim 4.5 \AA$ there might be an ET with zero activation energy, the overall process still may have an energy barrier arising from the partial desolvation. These arguments seem to reinforce the hypothesis of an ion transfer reaction, in agreement with previous theoretical works concluding that the halide discharge on metallic electrodes takes place mainly as an ion transfer process. ${ }^{14,26}$ Nevertheless, the approximations in the present study do not permit us to firmly reject the hypothesis of an electron transfer process.

To summarize, an ab initio model has been presented that is able to capture the essential physics of the electron transfer process between an anion and an electrode under electrochemical conditions; this implies that solvent effects and external potential are included simultaneously. Nevertheless, it is also important to realize that the model has also some weak points. In particular, we note that the representation of the solute-solvent interactions in the quantum chemical calculations is only an approximation. Most of the electrostatic interactions have been included, although shortrange repulsion forces, polarization effects, and electronic interactions of the solvent molecules with the redox center and with the electrode have not been taken into account. In the view of the results of previous studies, ${ }^{17,32,33}$ their inclusion would have a minor effect on the distribution of the solvent molecules in the system. On the other hand, their presence can exert an influence in the energies computed for the electronic states involved in the process. While these effects may be important for a more quantitative description of electron transfer processes in electrochemical environment, it is very unlikely that these model refinements will substantially change the conclusions of the present study. 


\section{ACKNOWLEDGMENTS}

This research has been supported by Spanish Grant No. DGICYT BQU2002-04029-CO2-01 and, in part, by Generalitat de Catalunya Project No. 2001SGR-00043. Computer time was provided by the Centre de Supercomputació de Catalunya, CESCA, and Centre Europeu de Paral.lelisme de Barcelona, CEPBA. C.H. is indebted to the European Community for financial support through the IHP program under Contract No. HPRI-CT-1999-00071 held by the CESCA/ CEBPA. F.I. and D.D. are also grateful to the DURSI of the Generalitat de Catalunya for the additional financial support through the Distinció per a la promoció de la Recerca Universitària and for a predoctoral grant, respectively.

${ }^{1}$ A. M. Kuznetsov and J. Ulstrup, Electron Transfer in Chemistry and Biology: An Introduction to the Theory, Wiley Series in Theoretical Chemistry (Wiley, New York, 1998).

${ }^{2}$ M. D. Newton, Chem. Rev. (Washington, D.C.) 91, 767 (1991).

${ }^{3}$ R. A. Marcus and N. Sutin, Biochim. Biophys. Acta 811, 265 (1985).

${ }^{4}$ R. A. Marcus, J. Chem. Phys. 24, 966 (1956).

${ }^{5}$ R. A. Marcus, J. Chem. Phys. 43, 679 (1965).

${ }^{6}$ V. G. Levich, in Physical Chemistry, An Advanced Treatise, edited by H. Eyring, D. Henderson, and W. Jost (Academic, New York, 1970), Vol. 9B.

${ }^{7}$ R. A. Marcus, Annu. Rev. Phys. Chem. 15, 155 (1964).

${ }^{8}$ C. Hartnig and M. T. M. Koper, J. Chem. Phys. 115, 8540 (2001).

${ }^{9}$ R. B. Yelle and T. Ichiye, J. Phys. Chem. B 101, 4127 (1997).

${ }^{10}$ D. W. Small, D. V. Matyushov, and G. A. Voth, J. Am. Chem. Soc. 125, 7470 (2003).

${ }^{11}$ P. W. Anderson, Phys. Rev. 124, 41 (1961).

${ }^{12}$ D. M. Newns, Phys. Rev. 178, 1123 (1969).

${ }^{13}$ W. Schmickler, J. Electroanal. Chem. 204, 31 (1986).

${ }^{14}$ W. Schmickler, Electrochim. Acta 41, 2329 (1995).

${ }^{15}$ J. B. Straus and G. A. Voth, J. Phys. Chem. 97, 7388 (1993).

${ }^{16}$ A. Calhoun and G. A. Voth, J. Phys. Chem. 100, 10746 (1996).
${ }^{17}$ A. Calhoun and G. A. Voth, J. Electroanal. Chem. 450, 253 (1998).

${ }^{18}$ N. S. Hush, J. Electroanal. Chem. 470, 170 (1999).

${ }^{19}$ C. F. Zinola, A. J. Arvia, G. L. Estiu, and E. A. Castro, J. Phys. Chem. 98, 7566 (1994)

${ }^{20}$ Z. Nagy, L. A. Curtis, N. C. Hung, D. J. Zurawski, and R. M. Yonco, J. Electroanal. Chem. 325, 313 (1992).

${ }^{21}$ R. R. Nazmutdinov, G. A. Tsirlina, O. A. Petrii, Y. L. Kharkats, and A. M. Kuznetsov, Electrochim. Acta 45, 3521 (2000).

${ }^{22}$ A. B. Anderson and D. B. Kang, J. Phys. Chem. A 102, 5993 (1998).

${ }^{23}$ A. B. Anderson and T. V. Albu, J. Am. Chem. Soc. 121, 11855 (1999).

${ }^{24}$ T. V. Albu and A. B. Anderson, Electrochim. Acta 46, 3001 (2001).

${ }^{25}$ D. Domínguez-Ariza, C. Sousa, and F. Illas, J. Phys. Chem. B 106, 12483 (2002).

${ }^{26}$ C. Hartnig and M. T. M. Koper, J. Am. Chem. Soc. 125, 9840 (2003).

${ }^{27}$ H. J. C. Berendsen, J. R. Grigera, and T. P. Straatsma, J. Phys. Chem. 91, 6269 (1987).

${ }^{28}$ A. Ignaczak, J. A. N. F. Gomes, and S. Romanowski, J. Electroanal. Chem. 459, 175 (1998).

${ }^{29}$ A. Ignaczak and J. A. N. F. Gomes, J. Mol. Struct.: THEOCHEM 464, 227 (1999).

${ }^{30}$ S. H. Lee and J. C. Rasaiah, J. Phys. Chem. 100, 1420 (1996).

${ }^{31}$ I.-C. Yeh and M. L. Berkowitz, J. Chem. Phys. 111, 3155 (1999).

${ }^{32}$ S. Izvekov, A. Mazzolo, K. VanOpdorp, and G. A. Voth, J. Chem. Phys. 114, 3248 (2001).

${ }^{33}$ S. Izvekov and G. A. Voth, J. Chem. Phys. 115, 7196 (2001).

${ }^{34}$ P. J. Hay and W. R. Wadt, J. Chem. Phys. 82, 299 (1985).

${ }^{35}$ F. Illas, J. Rubio, and J. C. Barthelat, Chem. Phys. Lett. 119, 397 (1985).

${ }^{36}$ K. Pierloot, B. Dumez, P.-O. Widmark, and B. O. Roos, Theor. Chim. Acta 90, 87 (1995).

${ }^{37}$ J. O'M Bockris and A. K. N. Reddy, Modern Electrochemistry, Vol. 2 (Plenum, New York, 1976), Vol. 2.

${ }^{38}$ M. J. Weaver, Appl. Surf. Sci. 67, 147 (1993).

${ }^{39} \mathrm{~K}$. Andersson, M. Barysz, A. Bernhardsson et al., MOLCAS version 5.2, University of Lund, Sweden, 2000.

${ }^{40}$ D. A. Rose and I. Benjamin, J. Chem. Phys. 95, 6856 (1991).

${ }^{41}$ M. García-Hernández, P. S. Bagus, and F. Illas, Surf. Sci. 409, 69 (1998).

${ }^{42}$ D. A. Rose and I. Benjamín, Chem. Phys. Lett. 234, 209 (1995). 